\title{
Synthesis of galanthamine
}

\section{Laszlo Czollner ${ }^{a}$, Matthias Treub ${ }^{b}$ Johannes Froehlich ${ }^{b}$, Bernhard Kueenburg ${ }^{a}$, and Ulrich Jordis*b}

${ }^{a}$ Sanochemia Pharmazeutika AG, 2491 Neufeld, Austria ${ }^{b}$ Institute of Organic Chemistry, Vienna University of Technology, Getreidemarkt 9, 1060 Vienna, Austria

E-mail: ujordis@pop.tuwien.ac.at

(received 20 Feb 01; accepted 13 Nov 01; published on the web 21 Nov 01)

\begin{abstract}
The synthesis of $( \pm)$ - and (-)-galanthamine via 3,4-dihydro-6,7-dimethoxy-4'-oxo-spiro[5H]-2benzazepine-5,1'-[2]cyclohexene]-2(1H)-carboxylic acid ethyl ester (2) is described.
\end{abstract}

Keywords: Narwedine, apogalanthamine enone-dienone conversion, O-demethylation

\section{Introduction}

In the course of our numerous investigations into the industrial synthesis of galanthamine, ${ }^{1}$ we also considered using the known ${ }^{2}$ 3,4-dihydro-6,7-dimethoxy-4'-oxo-spiro[5H]-2-benzazepine5,1'-[2]cyclohexene]-2(1H)-carboxylic acid ethyl ester (2) as the key intermediate.

\section{Results and Discussion}

Compound 2 was brominated using either dibromo Meldrum's acid (method A) or benzyl trimethyl ammonium tribromide (method B) to produce a mixture of mono- and dibrominated spiro compounds $\mathbf{3 a}$ and $\mathbf{3 b}$. The conditions described in method B are those that gave the highest yield of pure 3a after optimized efforts. The mono- and dibrominated species were separated by column chromatography and converted to dienones 4 . As an alternative, hydrogen bromide was eliminated from the crude mixture of $\mathbf{3 a}$ and $\mathbf{3} \mathbf{b}$, and $\mathbf{4 a}$ was isolated by chromatography. Various reagent systems (boron tribromide, boron tribromide / ethyl sulfide, aluminum chloride / pyridine, and aluminum chloride / ethyl sulfide) were tested for the Odemethylation of 4a to initiate the intramolecular Michael addition and the formation of the skeleton of the narwedine type enone 5a. Under these reaction conditions, the principal products were identified as apogalanthamine derivatives 10a or 10b (see Scheme 3). 5a was obtained in $5 \%$ yield using $\mathrm{AlCl}_{3} / \mathrm{Et}_{2} \mathrm{~S}$ in methylene chloride. and converted to $( \pm)$-galanthamine $\left.(\mathbf{(} \pm)-\mathbf{1}\right)$ by 
methylation and successive L-Selectride and lithium aluminum hydride reductions. As another alternative, the intermediate 7c was prepared starting with narwedin derivative 6, now available in large quantities due to successful industrial synthesis ${ }^{1}$. L-Selectride reduction with concomitant $\mathrm{N}$-deformylation produced $\mathbf{7 a}$, which was debrominated to $\mathbf{7 b}$ and, reacted with ethyl chloroformate, yielded 7c (see Scheme 1).<smiles>CCON1CCC2(C=CC(=O)CC2)c2c(ccc(OC)c2OC)C1</smiles>

a: $27 \% \mathbf{3 a}$<smiles>CC=[13CH]C</smiles>

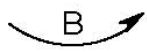

b: $53 \% 3 a$ $24 \% \mathbf{3 b}$

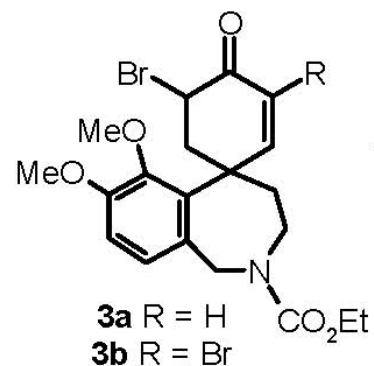

3b $\mathrm{R}=\mathrm{Br}$

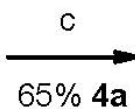

$34 \% \mathbf{4 b}$
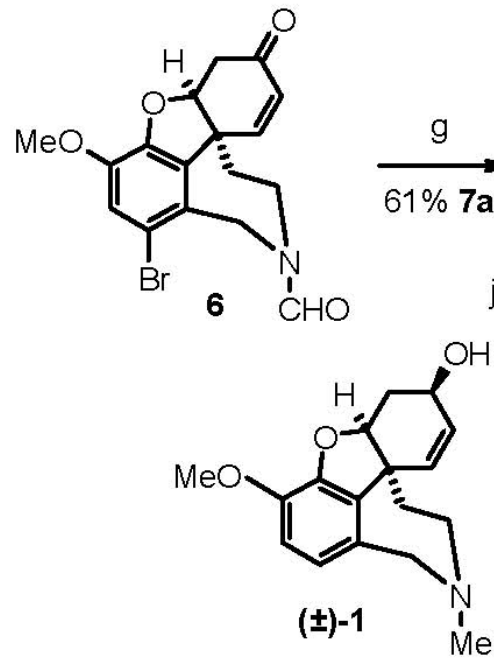

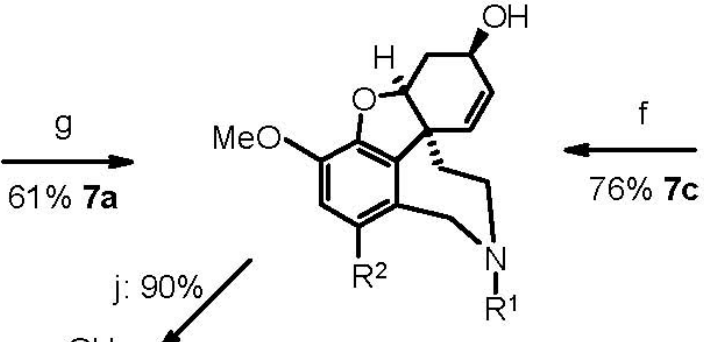

$76 \% 7 c$

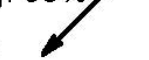

7a $R^{1}=H, R^{2}=B r$

7b $R^{1}=R^{2}=H$

7c R $\mathrm{R}^{1}=\mathrm{CO}_{2} \mathrm{Et}, \mathrm{R}^{2}=\mathrm{H}$ h: $82 \%$

i: $92 \%$

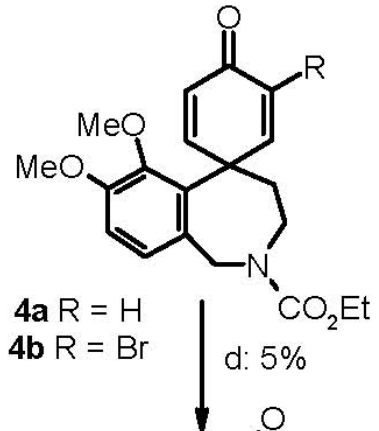

RO
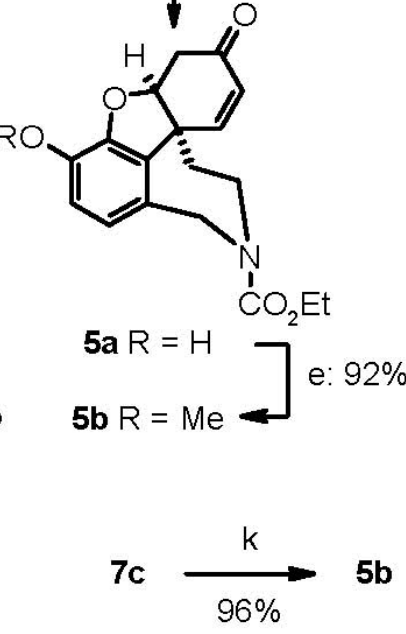

(a) 5,5-Dibromo-2,2-dimethyl-4,6-dioxo-1,3-dioxane, $\mathrm{CCl}_{4}$, reflux; (b) $\mathrm{BnMe}_{3} \mathrm{~N}^{+} \mathrm{Br}_{3}{ }^{-}, \mathrm{THF}$, rt;

(c) $\mathrm{DBN}, \mathrm{CHCl}_{3}, \mathrm{rt}$; (d) $\mathrm{Et}_{2} \mathrm{~S}, \mathrm{AlCl}_{3}, \mathrm{CH}_{2} \mathrm{Cl}_{2}$, rt; (e) $\mathrm{MeI}, \mathrm{K}_{2} \mathrm{CO}_{3}$, acetone, reflux; (f) LSelectride, THF, - $20^{\circ} \mathrm{C}$; (g) L-Selectride, THF, - $20^{\circ} \mathrm{C}$; (h) $\mathrm{CuZn}, \mathrm{CaCl}_{2}$, EtOH, water, reflux; (i) $\mathrm{ClCO}_{2} \mathrm{Et}, \mathrm{Et}_{3} \mathrm{~N}, \mathrm{CH}_{2} \mathrm{Cl}_{2}, 0^{\circ} \mathrm{C}$; (j) $\mathrm{LiAlH}_{4}, \mathrm{THF}$, reflux; (k) oxalyl chloride, DMSO, Et ${ }_{3} \mathrm{~N}$, $78^{\circ} \mathrm{C}$

\section{Scheme 1}

To synthesize (-)-galanthamine ((-)-1), 5b was converted to $( \pm)$-narwedine $(( \pm)-9)$ which was subjected to the crystallization induced chiral transformation ${ }^{3,4,5}$ to (-)-narwedine ((-)-9), followed by stereoselective L-Selectride reduction (see Scheme 2). 


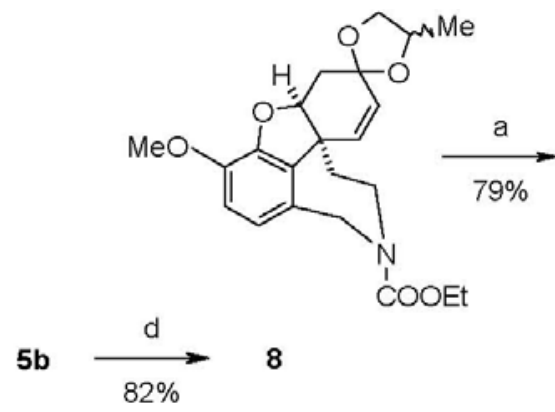

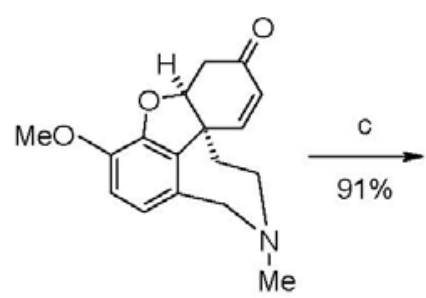

$(-)-9]$ b: $80 \%$

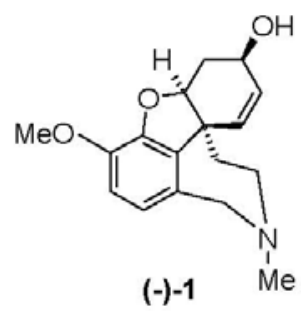

$(-)-1$

(a) $\mathrm{LiAlH}_{4}, \mathrm{THF}, 60{ }^{\circ} \mathrm{C}$, then acid workup; (b) EtOH/Et $3 \mathrm{~N}$, reflux; (c) L-Selectride, $\mathrm{THF},-20{ }^{\circ} \mathrm{C}$; (d) propylene glycol, $\mathrm{TsOH}$, toluene, reflux

\section{Scheme 2}

To confirm the structure, $\mathbf{1 0 b}$ was converted to the known apogalanthamine derivative $\mathbf{1 0 \mathbf { d } ^ { 6 }}$ by methylation and reduction (see Scheme 3).

$4 a$

a: $45 \% 10 b$

b: $80 \% 10 b$

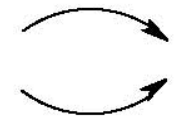

c: $67 \% 10 a$

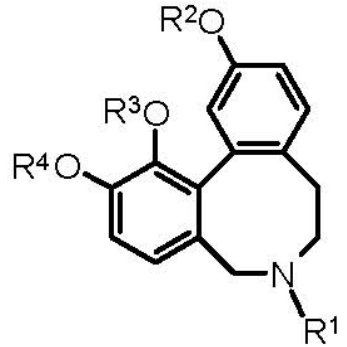

10a $\mathrm{R}^{1}=\mathrm{CO}_{2} \mathrm{Et}, \mathrm{R}^{2}=\mathrm{R}^{3}=\mathrm{R}^{4}=\mathrm{H}$

10b $R^{1}=\mathrm{CO}_{2} \mathrm{Et}, \mathrm{R}^{2}=\mathrm{H}, \mathrm{R}^{3}=\mathrm{R}^{4}=\mathrm{Me}$

10c $R^{1}=\mathrm{CO}_{2} E t, R^{2}=R^{3}=R^{4}=M e$
10d $R^{1}=R^{2}=R^{3}=R^{4}=M e$

(a) $\mathrm{AlCl}_{3}$, pyridine, $0{ }^{\circ} \mathrm{C}$; (b) $\mathrm{BBr}_{3}, \mathrm{Et}_{2} \mathrm{~S}, \mathrm{CH}_{2} \mathrm{Cl}_{2}, \mathrm{rt}$; (c) $\mathrm{BBr}_{3}, \mathrm{CH}_{2} \mathrm{Cl}_{2}, 0{ }^{\circ} \mathrm{C}$; (d) $\mathrm{MeI}, \mathrm{K}_{2} \mathrm{CO}_{3}$, acetone, reflux; (e) $\mathrm{LiAlH}_{4}, \mathrm{THF}, 0{ }^{\circ} \mathrm{C} \rightarrow \mathrm{rt}$

\section{Scheme 3}

\section{Experimental Section}

5'-Bromo-3,4-dihydro-6,7-dimethoxy-4'-oxo-spiro[5H-2-benzazepine-5,1'-[2]cyclohexene]2(1 H)-carboxylic acid, ethyl ester (3a). Method A. 5,5-Dibromo-2,2-dimethyl-4,6-dioxo-1,3dioxane (Dibromomeldrum's acid) $(120 \mathrm{mg}, 0.40 \mathrm{mmol})$ was added to the spiroenone 2 (140 $\mathrm{mg}$, $0.43 \mathrm{mmol})$ in $\mathrm{CCl}_{4}(10 \mathrm{~mL})$, and the reaction mixture was refluxed for $21 \mathrm{~h}$. After concentration the residue was purified by flash chromatography $\left(30 \mathrm{~g} \mathrm{SiO}_{2}\right.$, petrol ether : EtOAc, $80: 20$ to $60: 40)$ to give the monobrominated species $3 \mathbf{a}(50 \mathrm{mg}, 26.5 \%)$ and the dibromo product $3 \mathbf{b}$ (20 mg, 9.1\%) both as yellow oils.

Method B. Benzyltrimethylammonium tribromide (480 $\mathrm{mg}, 1.23 \mathrm{mmol}$ ) was added to the spiroenone $2(320 \mathrm{mg}, 6.89 \mathrm{mmol})$ in $\mathrm{THF}(5 \mathrm{~mL})$ at $0{ }^{\circ} \mathrm{C}$ within $30 \mathrm{~min}$, and the reaction mixture was stirred at room temperature for $3 \mathrm{~h} . \mathrm{CHCl}_{3}(30 \mathrm{~mL})$ was added and the organic layer was washed with a saturated aqueous $\mathrm{NaHCO}_{3}$ solution $(2 \times 40 \mathrm{~mL})$, dried $\left(\mathrm{Na}_{2} \mathrm{SO}_{4}\right)$, filtered and 
concentrated to give the crude mixture of products, which were separated by flash chromatography using the same eluents as described in method A to yield the monobromo product 3a (228 mg, 53\%) and dibrominated species $3 \mathbf{b}(20 \mathrm{mg}, 24 \%)$. Analytical data of 3a: ${ }^{1} \mathrm{H}$ NMR $\left(\mathrm{CDCl}_{3}\right) \delta 7.02(\mathrm{~d}, 1 \mathrm{H}), 7.20$ - $7.00(\mathrm{~m}, 2 \mathrm{H}), 6.05(\mathrm{~d}, 1 \mathrm{H}), 4.60-4.40(\mathrm{~m}, 2 \mathrm{H}), 4.30$ $3.95(\mathrm{~m}, 3 \mathrm{H}), 3.85(\mathrm{~s}, 3 \mathrm{H}), 3.68(\mathrm{~s}, 3 \mathrm{H}), 3.72(\mathrm{dd}, 1 \mathrm{H}), 3.60(\mathrm{~m}, 1 \mathrm{H}), 3.00(\mathrm{dd}, 1 \mathrm{H}), 2.65(\mathrm{~m}$, $1 \mathrm{H}), 2.35(\mathrm{~m}, 2 \mathrm{H}), 1.2(\mathrm{~m}, 3 \mathrm{H})$. Analytical data of $3 \mathbf{b}:{ }^{1} \mathrm{H}$ NMR $\left(\mathrm{CDCl}_{3}\right) \delta 7.10-6.85(\mathrm{~m}, 2$ H), $6.05(\mathrm{~d}, 1 \mathrm{H}), 4.90(\mathrm{dd}, 1 \mathrm{H}), 4.65(\mathrm{~m}, 1 \mathrm{H}), 4.40(\mathrm{~m}, 1 \mathrm{H}), 4.20-3.95(\mathrm{~m}, 2 \mathrm{H}), 3.88(\mathrm{~s}, 3$ H). $3.75(\mathrm{~s}, 3 \mathrm{H}), 3.50(\mathrm{~m}, 1 \mathrm{H}), 3.00(\mathrm{~m}, 1 \mathrm{H}), 2.65(\mathrm{~m}, 1 \mathrm{H}), 2.45(\mathrm{~m}, 1 \mathrm{H}), 2.15(\mathrm{~m}, 2 \mathrm{H}), 1.20$ (m, $3 \mathrm{H})$.

\section{3,4-Dihydro-6,7-dimethoxy-4'-oxo-spiro[5H-2-benzazepine-5,1'-[2,5]cyclohexa-diene]-}

2(1H)-carboxylic acid, ethyl ester (4a). Method B was performed on a $6.67 \mathrm{mmol}$ scale and the crude bromination product obtained in almost quantitative yield was dissolved in $\mathrm{CHCl}_{3}$ $(50 \mathrm{~mL})$, 1,5-diazabicylco[4.3.0]non-5-ene (DBN) $(2.1 \mathrm{~mL}, 17.6 \mathrm{mmol})$ was added and the reaction mixture was stirred ad room temperature for $16 \mathrm{~h} . \mathrm{CHCl}_{3}(30 \mathrm{~mL})$ was added, and the organic layer was washed with a saturated aqueous $\mathrm{NaHCO}_{3}$ solution (2 x $25 \mathrm{~mL}$ ), dried $\left(\mathrm{Na}_{2} \mathrm{SO}_{4}\right)$, filtered and concentrated. Flash chromatography (50 g SiO 2 , hexane : EtOAc, $60: 40$ to $50: 50)$ gave compound $4 \mathbf{a}$ as a yellow oil (1.55 g, 65\% for 2 steps) and bromodienone $4 \mathbf{b}$ $(1.0 \mathrm{~g}, 34 \%)$ as a colorless solid. Analytical data of 4a: IR (KBr): 3465 br, $2948.6 \mathrm{~m}, 1680.9 \mathrm{~s}$, $1663.4 \mathrm{~s}, 1525.4 \mathrm{w}, 1470.2 \mathrm{~m}, 1419.5 \mathrm{~m}, 1252.6 \mathrm{~m}, 1124.8 \mathrm{~m}, 1094.5 \mathrm{~m}, 1054.6 \mathrm{~m} ; 971.3 \mathrm{w}$, $759.3 \mathrm{~m} \mathrm{~cm}-1 ;{ }^{1} \mathrm{H}$ NMR $\left(\mathrm{CDCl}_{3}\right) \delta 7.46(\mathrm{~d}, 1 \mathrm{H}), 6.94(\mathrm{~m}, 2 \mathrm{H}), 6.81(\mathrm{~m}, 1 \mathrm{H}), 6.38(\mathrm{~d}, 1 \mathrm{H})$, $4.60(\mathrm{~d}, 1 \mathrm{H}), 4.13(\mathrm{~m}, 2 \mathrm{H}), 3.81(\mathrm{~s}, 3 \mathrm{H}), 3.69(\mathrm{~m}, 2 \mathrm{H}), 3.59(\mathrm{~s}, 3 \mathrm{H}), 2.26(\mathrm{~m}, 2 \mathrm{H}), 1.22(\mathrm{~m}, 3$ $\mathrm{H}) ;{ }^{13} \mathrm{C} \mathrm{NMR}\left(\mathrm{CDCl}_{3}\right) \delta 178.2(\mathrm{~s}), 154.9$ and $154.5(\mathrm{~s}), 154.1$ and $153.8(\mathrm{~d}), 153.2(\mathrm{~d}), 151.8(\mathrm{~s})$, $148.5(\mathrm{~s}), 130.4(\mathrm{~s}), 129.2(\mathrm{~s}), 125.4$ and 125.1 (d), 124.5 and $123.8(\mathrm{~d}), 121.9$ and $121.4(\mathrm{~s})$, 111.4 and $111.1(\mathrm{~d}), 61.2$ and $61.0(\mathrm{t}), 60.5(\mathrm{q}), 55.2(\mathrm{q}), 50.4(\mathrm{~s}), 47.3(\mathrm{t}), 43.6(\mathrm{t}), 37.5$ and 37.3 (t), 14.2 and 14.1 (q); MS (EI, $70 \mathrm{eV}, \mathrm{m} / \mathrm{e}): 438$ (2.3), 437 (15.1), 435 (114.8), 498 (14.6), 406 (16.0), 357 (19.1), 356 (100), 321 (10.5), 319 (10.6), 268 (17.6), 267 (53.2), 255 (49.8), 254 (43.8), 253 (10.3), 252 (20.0), 130 (9.5), 129 (28.5), 128 (37.5), 127 (23.3), 119 (17.7), 118 (32.5), 117 (29.5), 116 (10.1), 109 (18.8), 108 (20.8), 107 (12.9), 106 (13.3), 100 (15.5), 99 (30.2), 98 (25.7), 97 (19.4), 88 (18.8 ), 87 (17.1), 86 ((28.5), 85 (10.4 ), 76 (10.1), 75 (24.27), 74 (17.2), 73 (13.2), 72 (9.3), 71 (27.7). 62 (12.8), 61 (11.5), 60 (22.2), 59 (39.35), 58 (35.1), 57 (8.1), 45 (18.4), 43 (24.7); TLC: hexane : EtOAc $=3: 2, \mathrm{R}_{\mathrm{f}}=0.4$. Analytical data of 4b: IR (KBr): 3480 s, 2963 s, 1696 s, 1658 s, 1618 s, 1600 m, 1569 w, 1471 s, 1420 s, 1252 s, 1210 s, $1125 \mathrm{~m}, 1034 \mathrm{~m}, 1057 \mathrm{~m}, 1015 \mathrm{~m}, 969 \mathrm{w}, 939 \mathrm{~m}, 890 \mathrm{w}, 850 \mathrm{~m}, 807 \mathrm{w}, 757 \mathrm{~m}, 682 \mathrm{w} \mathrm{cm}^{-1}$; ${ }^{1} \mathrm{H}$ NMR $\left(\mathrm{CDCl}_{3}\right) \delta 7.00(\mathrm{~m}, 2 \mathrm{H}), 6.80(\mathrm{~m}, 2 \mathrm{H}), 6.28(\mathrm{~m}, 2 \mathrm{H}), 4.60(\mathrm{~d}, 2 \mathrm{H}), 4.08(\mathrm{~m}, 2 \mathrm{H}), 3.80$ $(\mathrm{s}, 3 \mathrm{H}), 3.68(\mathrm{~m}, 2 \mathrm{H}), 3.55(\mathrm{~s}, 3 \mathrm{H}), 2.20(\mathrm{t}, 2 \mathrm{H}), 1.15(\mathrm{~m}, 3 \mathrm{H}) ;{ }^{13} \mathrm{C} \mathrm{NMR}\left(\mathrm{CDCl}_{3}\right) \delta 185.6(\mathrm{~s})$, 155.4 (s), 154.5 (d), 154.5 (d), 154.5 (s), 152.1 (s), 148.9 (s), 130.9 and 130.7 (s), 125.8 (d), $125.8(\mathrm{~d}), 125.5$ and $125.2(\mathrm{~d}), 111.3$ and $111.0(\mathrm{~d}), 61.1(\mathrm{t}), 60.4(\mathrm{q}), 55.4(\mathrm{q}), 47.5(\mathrm{~s}), 47.5(\mathrm{t})$, $43.9(\mathrm{t}), 38.2$ and $38.1(\mathrm{t}), 14.5$ and $14.3(\mathrm{q})$.

5,6,9,10,11,12-Hexahydro-3-hydroxy-6-oxo-4aH-benzofuro[3a,3,2-ef][2]benzazepin-11carboxylic acid ethyl ester (5a). Dienone 4 a $(200 \mathrm{mg}, 0.61 \mathrm{mmol})$ in $\mathrm{CH}_{2} \mathrm{Cl}_{2}(5 \mathrm{~mL})$ was added 
to a solution of $\mathrm{AlCl}_{3}$ (freshly sublimed, $300 \mathrm{mg}, 2.24 \mathrm{mmol}$ ) and diethyl sulfide (1.4 mL, $13.2 \mathrm{mmol})$ in $\mathrm{CH}_{2} \mathrm{Cl}_{2}(10 \mathrm{~mL})$ at room temperature and stirred for $4 \mathrm{~h}$. A mixture of $\mathrm{MeOH}$ $(4 \mathrm{~mL})$, water $(1 \mathrm{~mL})$ and $\mathrm{CH}_{2} \mathrm{Cl}_{2}(5 \mathrm{~mL})$ was added, and the organic layer was washed with water $(1 \times 20 \mathrm{~mL})$. The aqueous layer was extracted with $\mathrm{CH}_{2} \mathrm{Cl}_{2}(3 \times 15 \mathrm{~mL})$, the combined organic layers were washed with brine $(1 \times 30 \mathrm{~mL})$, dried $\left(\mathrm{Na}_{2} \mathrm{SO}_{4}\right)$, filtered and concentrated. Flash chromatography $\left(20 \mathrm{~g} \mathrm{SiO}_{2}\right.$, hexane : EtOAc $\left.=60: 40\right)$ gave compound 5a as a colorless oil (10 mg, 5.2\%). IR (KBr): 3454 br, 2942 m, 2938 m, 2935 s, 1694 s, 1617 s, 1490 s, 1427 s, $1240 \mathrm{~s}, 1101 \mathrm{~m}, 1067 \mathrm{~m}, 1031 \mathrm{~m}, 973 \mathrm{~m}, 947 \mathrm{~m}, 917 \mathrm{~m}, 851 \mathrm{~m}, 772 \mathrm{~m}, 699 \mathrm{w} \mathrm{cm}^{-1}$; ${ }^{1} \mathrm{H}$ NMR $\left(\mathrm{CDCl}_{3}\right) \delta 6.95(\mathrm{dd}, 1 \mathrm{H}), 6.75(\mathrm{~m}, 2 \mathrm{H}), 6.03(\mathrm{~d}, 1 \mathrm{H}), 5.60(\mathrm{~s}, \mathrm{OH}), 4.90(\mathrm{dd}, 1 \mathrm{H}), 4.62(\mathrm{~d}, 1$ H), $4.40(\mathrm{~d}, 1 \mathrm{H}), 4.20-3.90(\mathrm{~m}, 3 \mathrm{H}), 3.35(\mathrm{~m}, 1 \mathrm{H}), 3.10(\mathrm{~m}, 1 \mathrm{H}), 2.75(\mathrm{dd}, 1 \mathrm{H}), 2.28-1.92$ $(\mathrm{m}, 2 \mathrm{H}), 1.22(\mathrm{~m}, 3 \mathrm{H})$; TLC: hexane : EtOAc $=3: 2, \mathrm{R}_{\mathrm{f}}=0.4$.

5,6,7,8-Tetrahydro-11-hydroxy-1,2-dimethoxydibenz[c,e]azocine-6-carboxylic acid ethyl ester (10b). Method A. Dienone 4a (100 mg, $0.31 \mathrm{mmol})$ in $\mathrm{CH}_{2} \mathrm{Cl}_{2}(3 \mathrm{~mL})$ was added to a solution of $\mathrm{BBr}_{3}(228 \mathrm{mg}, 0.91 \mathrm{mmol})$ and diethyl sulfide $(279 \mathrm{mg}, 3.10 \mathrm{mmol})$ in $\mathrm{CH}_{2} \mathrm{Cl}_{2}$ $(5 \mathrm{~mL})$ at room temperature and stirred for $20 \mathrm{~min}$. A saturated aqueous $\mathrm{NaHCO}_{3}$ solution $(80 \mathrm{~mL})$ was added, the layers were separated and the aqueous layer was extracted with $\mathrm{CH}_{2} \mathrm{Cl}_{2}$ $(3 \times 20 \mathrm{~mL})$. The combined organic layers were washed with brine $(1 \times 30 \mathrm{~mL})$, dried $\left(\mathrm{Na}_{2} \mathrm{SO}_{4}\right)$, filtered and concentrated. Flash chromatography $\left(20 \mathrm{~g} \mathrm{SiO}_{2}\right.$, hexane - EtOAc, $70: 30$ to $\left.60: 40\right)$ yielded compound 10b as colorless oil (80 $\mathrm{mg}, 80 \%)$.

Method B. Dienone 4a (178 mg, $0.55 \mathrm{mmol}$ ) was added to a solution of $\mathrm{AlCl}_{3}$ (freshly sublimated, $2.30 \mathrm{~g}, 17.2 \mathrm{mmol})$ in pyridine $(14 \mathrm{~mL})$ at $0{ }^{\circ} \mathrm{C}$ and stirred at room temperature for $28 \mathrm{~h}$. After concentration the residue was dissolved in $\mathrm{CHCl}_{3}(80 \mathrm{~mL})$ and washed with $2 \mathrm{~N} 2 \mathrm{~N}$ $\mathrm{HCl}(1 \times 80 \mathrm{~mL})$. The aqueous layer was extracted with $\mathrm{CHCl}_{3}(2 \times 20 \mathrm{~mL})$, the combined organic layers were washed with brine $(1 \times 30 \mathrm{~mL})$, dried $\left(\mathrm{Na}_{2} \mathrm{SO}_{4}\right)$, filtered and concentrated. Flash chromatography $\left(20 \mathrm{~g} \mathrm{SiO}_{2}\right.$, hexane : EtOAc, $70: 30$ to $\left.60: 40\right)$ yielded compound 10b as colorless oil $(80 \mathrm{mg}, 54 \%) .{ }^{1} \mathrm{H}$ NMR $\left(\mathrm{CDCl}_{3}\right) \delta 7.55$ and $7.32(\mathrm{~d}, 1 \mathrm{H}), 7.15(\mathrm{~m}, 1 \mathrm{H}), 6.90(\mathrm{~d}, 1$ $\mathrm{H}), 6.78(\mathrm{~m}, 2 \mathrm{H}), 6.30(\mathrm{~d}, \mathrm{OH}), 4.80(\mathrm{dd}, 1 \mathrm{H}), 4.62-4.30(\mathrm{~m}, 1 \mathrm{H}), 4.25-4.00(\mathrm{~m}, 2 \mathrm{H}), 3.95$ $(\mathrm{s}, 3 \mathrm{H}), 3.48(\mathrm{~s}, 3 \mathrm{H}), 3.20(\mathrm{t}, 1 \mathrm{H}), 2.95(\mathrm{t}, 1 \mathrm{H}), 2.80(\mathrm{~m}, 1 \mathrm{H}), 1.84(\mathrm{dd}, 1 \mathrm{H}), 1.50-1.15$ (m, 3 $\mathrm{H}) ;{ }^{13} \mathrm{C} \mathrm{NMR}(\mathrm{CDCl} 3) \delta 156.1 \mathrm{~s}, 155.9(\mathrm{~s}), 152.2$ and 152.1 (s), 146.2 and 145.9 (s), 141.7 and $141.5(\mathrm{~s}), 134.5$ and 134.1 (s), 132.2 (d), 130.9 and 130.8 (s), 127.3 and 126.9 (s), 126.3 and $125.7(\mathrm{~d}), 115.5(\mathrm{~d}), 113.1$ (d), 111.3 and $111.2(\mathrm{~d}), 61.5$ and $61.4(\mathrm{t}), 60.3(\mathrm{q}), 55.7$ (q), 47.5 and $47.2(\mathrm{t}), 47.1$ and $46.9(\mathrm{t}), 34.9$ and $34.8(\mathrm{t}), 14.7$ and $14.5(\mathrm{q})$; TLC: hexane - EtOAc $=3: 2, \mathrm{R}_{\mathrm{f}}$ $=0.3$.

5,6,7,8-Tetrahydro-1,2,11-trimethoxydibenz[c,e]azocine-6-carboxylic acid ethyl ester (10c). To a suspension of $\mathrm{K}_{2} \mathrm{CO}_{3}(1.30 \mathrm{~g}, 9.40 \mathrm{mmol})$ in acetone $(30 \mathrm{~mL})$ phenol $10 \mathrm{~b}(310 \mathrm{mg}$, $0.86 \mathrm{mmol}$ ) an methyl iodide $(681 \mathrm{mg}, 4.80 \mathrm{mmol})$ were added, and the mixture was refluxed for $15 \mathrm{~h}$. After concentration water $(60 \mathrm{~mL})$ was added to the residue. The aqueous phase was extracted with EtOAc $(3 \times 30 \mathrm{~mL})$, the combined organic layers were washed with brine $(1 \mathrm{x}$ $50 \mathrm{~mL})$, dried $\left(\mathrm{Na}_{2} \mathrm{SO}_{4}\right)$, filtered and concentrated. Flash chromatography $\left(60 \mathrm{~g} \mathrm{SiO}_{2}\right.$, hexane : EtOAc, 60 : 40) yielded compound 10c as colorless oil (304 mg, 94\%). ${ }^{1} \mathrm{H} \mathrm{NMR}\left(\mathrm{CDCl}_{3}\right) \delta 7.55$ 
and $7.35(\mathrm{~d}, 1 \mathrm{H}), 7.25(\mathrm{~m}, 1 \mathrm{H}), 6.95-6.75(\mathrm{~m}, 3 \mathrm{H}), 5.00-4.70(\mathrm{dd}, 1 \mathrm{H}), 4.65-4.45(\mathrm{~m}, 1$ H), $4.40-4.00(\mathrm{~m}, 2 \mathrm{H}), 3.91(\mathrm{~s}, 3 \mathrm{H}), 3.86(\mathrm{~s}, 3 \mathrm{H}), 3.42(\mathrm{~s}, 3 \mathrm{H}), 3.13(\mathrm{t}, 1 \mathrm{H}), 2.85(\mathrm{~m}, 2 \mathrm{H})$, $2.40(\mathrm{dd}, 1 \mathrm{H}), 1.50-1.20(\mathrm{~m}, 3 \mathrm{H}) ;{ }^{13} \mathrm{C} \mathrm{NMR}\left(\mathrm{CDCl}_{3}\right) \delta 159.4(\mathrm{~s}), 155.7$ (s), 152.2 and 152.2 (s), 146.3 and 146.1 (s), 141.8 and 141.5 (s), 134.5 and 134.1 (s), 132.2 (d), 131.1 and 131.0 (s), 127.7 and $127.5(\mathrm{~s}), 126.4$ and $125.7(\mathrm{~d}), 114.3$ and $114.1(\mathrm{~d}), 111.4(\mathrm{~d}), 111.3(\mathrm{~d}), 61.3(\mathrm{t}), 60.3$ (q), $55.8(\mathrm{q}), 55.1(\mathrm{q}), 47.5(\mathrm{t}), 47.2$ and $47.0(\mathrm{t}), 35.2(\mathrm{t}), 14.8$ and $14.6(\mathrm{q})$.

5,6,7,8-Tetrahydro-1,2,11-trihydroxydibenz[c,e]azocine-6-carboxylic acid ethyl ester (10a). Dienone 4a $(250 \mathrm{mg}, 0.78 \mathrm{mmol})$ in $\mathrm{CH}_{2} \mathrm{Cl}_{2}(5 \mathrm{~mL})$ was added to a solution of $\mathrm{BBr}_{3}(876 \mathrm{mg}$, $3.50 \mathrm{mmol})$ in $\mathrm{CH}_{2} \mathrm{Cl}_{2}(10 \mathrm{~mL})$ at $0{ }^{\circ} \mathrm{C}$ and stirred for $60 \mathrm{~min}$ at this temperature. Water $(10 \mathrm{~mL})$ was added and the $\mathrm{pH}$ was adjusted to 9 using $2 \mathrm{~N} \mathrm{NaOH}$. The layers were separated and the aqueous layer was acidified with $2 \mathrm{~N} \mathrm{HCl}$ and extracted with $\mathrm{CHCl}_{3}(3 \times 50 \mathrm{~mL})$. The combined organic layers were washed with brine $(1$ x $50 \mathrm{~mL})$, dried $\left(\mathrm{Na}_{2} \mathrm{SO}_{4}\right)$, filtered and concentrated. Flash chromatography (22 $\mathrm{g} \mathrm{SiO}_{2}$, hexane : EtOAc, $70: 30$ to $60: 40$ to $50: 50$ ) yielded compound 10a as colorless solid (155 mg, 67\%). IR (KBr): 3307 br, 1633 s, 1470 s, $1440 \mathrm{~s}, 1408$ $\mathrm{m}, 1270 \mathrm{~s}, 1288 \mathrm{~s}, 1113 \mathrm{~s}, 1075 \mathrm{~m}, 1026 \mathrm{w}, 991 \mathrm{~m}, 910 \mathrm{w}, 906 \mathrm{w}, 790 \mathrm{w}, 610 \mathrm{w}$, $590 \mathrm{w}, 418 \mathrm{~cm}^{-1} ;{ }^{1} \mathrm{H}$ NMR (DMSO-d 6$) \delta 8.65(\mathrm{~s}, \mathrm{OH}), 8.43(\mathrm{~s}, \mathrm{OH}), 6.95-6.40$ $(\mathrm{m}, 5 \mathrm{H}), 6.25(\mathrm{~s}, \mathrm{OH}), 4.45(\mathrm{dd}, 1 \mathrm{H}), 4,35-3.98(\mathrm{~m}, 1 \mathrm{H}), 3.95-3.70(\mathrm{~m}, 2 \mathrm{H}), 2.85(\mathrm{t}, 1$ H), $2.50(\mathrm{~m}, 2 \mathrm{H}), 2.05(\mathrm{t}, 1 \mathrm{H}), 1.20-0.90(\mathrm{~m}, 3 \mathrm{H}) ;{ }^{13} \mathrm{C}$ NMR (DMSO-d 6$) \delta 156.7$ (s), 155.1 (s), 143.9 and 143.7 (s), 141.5 (s), 141.2 and 141.1 (s), 131.6 (d), 129.3 and 129.3 (s), 127.1 and $126.9(\mathrm{~s}), 125.5$ and $125.7(\mathrm{~s}), 121.3$ and $120.9(\mathrm{~d}), 115.4(\mathrm{~d}), 113.7(\mathrm{~d}), 112.8(\mathrm{~d}), 60.6(\mathrm{t}), 46.5$ and $46.7(\mathrm{t}), 46.8$ and $47.0(\mathrm{t}), 34.5(\mathrm{t}), 14.4$ and $14.7(\mathrm{q})$; TLC: hexane : EtOAc $=3: 2, \mathrm{R}_{\mathrm{f}}=0.1$; m.p.: $213-218^{\circ} \mathrm{C}$.

5,6,7,8-Tetrahydro-1,2,11-trimethoxy-6-methyl-dibenz[c,e]azocine (10d). Carbamate 10c (175 mg, $0.47 \mathrm{mmol})$ was added to a suspension of $\mathrm{LiAlH}_{4}(39 \mathrm{mg}, 1.02 \mathrm{mmol})$ in anhydrous THF $(10 \mathrm{~mL})$ at $0{ }^{\circ} \mathrm{C}$ and stirred at room temperature overnight. Water $(100 \mathrm{~mL})$ was added, and the aqueous layer was extracted with EtOAc $(4 \times 100 \mathrm{~mL})$. The combined organic layers were washed with brine $(1 \times 50 \mathrm{~mL})$, dried $\left(\mathrm{Na}_{2} \mathrm{SO}_{4}\right)$, filtered and concentrated. Flash chromatography (30 $\mathrm{g} \mathrm{SiO}_{2}$, EtOAc : hexane, $90: 10$ ) gave compound 10d as colorless oil (90 mg, 61\%). m.p. $165-169{ }^{\circ} \mathrm{C}$ (perchlorate, Lit. $\left.{ }^{6}: 167-170{ }^{\circ} \mathrm{C}\right) ;{ }^{1} \mathrm{H}$ NMR $\left(D_{M S O}-d_{6}\right) \delta 7.28(\mathrm{~d}, 1 \mathrm{H}), 7.10(\mathrm{~d}, 1$ H), $6.95(\mathrm{~d}, 1 \mathrm{H}), 6.65(\mathrm{~m}, 2 \mathrm{H}), 3.90(\mathrm{~s}, 3 \mathrm{H}), 3.85(\mathrm{~s}, 3 \mathrm{H}), 3.50(\mathrm{~d}, 1 \mathrm{H}), 3.42(\mathrm{~s}, 3 \mathrm{H}), 3.20$ (d, $1 \mathrm{H}), 3.00(\mathrm{~d}, 1 \mathrm{H}), 2.70-2.35(\mathrm{~m}, 3 \mathrm{H}), 3.40(\mathrm{~s}, 3 \mathrm{H}) ;{ }^{13} \mathrm{C}$ NMR $\left(\mathrm{DMSO}-\mathrm{d}_{6}\right) \delta 158.8(\mathrm{~s}), 151.3$ (s), 145.7 (s), 142.3 (s), 133.6 (s), 131.2 (s), 131.0 (d), 127.1 (s), 125.6 (d), 113.5 (d), 110.8 (d), 110.4 (d), 59.7 (q), 58.2 (q), 57.0 (t), 55.3 (t), 54.5 (q), 44.8 (q), 32.0 (t); TLC: EtOAc : Et3N = $95: 5, \mathrm{R}_{\mathrm{f}}=0.3$.

\section{4a,5,9,10-Tetrahydro-3-methoxy-6-oxo-6H-benzofuro[3a,3,2-ef][2]benzazepine-11(12H)-} carboxylic acid ethyl ester (5b). To a suspension of $\mathrm{K}_{2} \mathrm{CO}_{3}(0.50 \mathrm{~g}, 6.62 \mathrm{mmol})$ in acetone $(20 \mathrm{~mL})$ phenol $5 a(100 \mathrm{mg}, 0.29 \mathrm{mmol})$ and methyl iodide $(454 \mathrm{mg}, 3.20 \mathrm{mmol})$ were added, and the mixture was refluxed for $6 \mathrm{~h}$. After concentration water $(60 \mathrm{~mL})$ was added to the residue. The aqueous phase was extracted with EtOAc $(3 \times 30 \mathrm{~mL})$, the combined organic layers were washed with brine $(1 \times 50 \mathrm{~mL})$, dried $\left(\mathrm{Na}_{2} \mathrm{SO}_{4}\right)$, filtered and concentrated. Flash 
chromatography (50 $\left.\mathrm{g} \mathrm{SiO}_{2}, \mathrm{CHCl}_{3}: \mathrm{MeOH}, 99: 1\right)$ yielded compound $\mathbf{5 b}$ as colorless solid (65 mg, 65\%). IR (KBr): 3441 br, 2975 m, 2902 m, 1699 s, 1624 m, 1511 s, 1432 s, 1289 s, 1249 s, 1217s, 1194 s, 1139 m, 1107 m, 1057 m, 1020 m, 993 m, 930 w, 880 w, 837 w, 809 w, 775 $\mathrm{m} \mathrm{cm}^{-1} ;{ }^{1} \mathrm{H}$ NMR $\left(\mathrm{CDCl}_{3}\right) \delta 6.85(\mathrm{~d}, 1 \mathrm{H}), 6.90-6.60(\mathrm{~m}, 2 \mathrm{H}), 6.03(\mathrm{~d}, 1 \mathrm{H}), 4.90(\mathrm{dd}, 1 \mathrm{H})$, $4.70(\mathrm{~s}, 1 \mathrm{H}), 4.30(\mathrm{dd}, 1 \mathrm{H}), 4.20-3.90(\mathrm{~m}, 3 \mathrm{H}), 3.80(\mathrm{~s}, 3 \mathrm{H}), 3.35(\mathrm{~m}, 1 \mathrm{H}), 3.15(\mathrm{dd}, 1 \mathrm{H})$, $2.75(\mathrm{dd}, 1 \mathrm{H}), 2.28-1.90(\mathrm{~m}, 2 \mathrm{H}), 1.20(\mathrm{t}, 3 \mathrm{H}) ;{ }^{13} \mathrm{C} \mathrm{NMR}\left(\mathrm{CDCl}_{3}\right) \delta 193.7(\mathrm{~s}), 155.1$ and $155.0(\mathrm{~s}), 147.2$ (s), 143.8 (s), 143.3 and 143.2 (d), 129.5 and 129.2 (s), 129.0 (s), 126.9 (d), 121.1 and $120.6(\mathrm{~d}), 111.5$ and $111.3(\mathrm{~d}), 87.3(\mathrm{~d}), 61.1(\mathrm{t}), 55.6(\mathrm{q}), 51.4$ and $51.0(\mathrm{t}), 48.7(\mathrm{~s})$, 45.5 and $45.2(\mathrm{t}), 36.8(\mathrm{t}), 36.1$ and $35.2(\mathrm{t}), 14.2$ and $14.1(\mathrm{q})$; TLC: $\mathrm{CHCl}_{3}: \mathrm{MeOH}=99: 1, \mathrm{R}_{\mathrm{f}}$ $=0.4 ; \mathrm{m} . \mathrm{p} .: 154-156^{\circ} \mathrm{C}$.

(6R)-1-Bromo-3-methoxy-5,6,9,10,11,12-hexahydro-4aH-benzofuro[3a,3,2ef][2]benzazepin-6-ol (7a). To a suspension of ketone 6 (15.0 g, $39.7 \mathrm{mmol})$ in dry THF $(400 \mathrm{~mL})$ L-Selectride ${ }^{\circledR}(120 \mathrm{~mL}, 1 \mathrm{~N}$ in THF $)$ was added at $-20^{\circ} \mathrm{C}$. The mixture was stirred for $2 \mathrm{~h}$ at this temperature, then water $(100 \mathrm{~mL})$ was added dropwise. After stirring for $10 \mathrm{~min}$. the mixture was concentrated, and the residue was dissolved in EtOAc $(300 \mathrm{~mL})$. The organic layer was washed with a saturated aqueous $\mathrm{NaHCO}_{3}$ solution $(1 \times 300 \mathrm{~mL})$, the aqueous layer was extracted with EtOAc $(2 \times 100 \mathrm{~mL})$. The combined organic layers were washed with a saturated aqueous $\mathrm{NaHCO}_{3}$ solution $(2 \times 300 \mathrm{~mL})$, water $(1 \times 100 \mathrm{~mL})$, brine $(1 \times 300 \mathrm{~mL})$, dried $\left(\mathrm{Na}_{2} \mathrm{SO}_{4}\right)$, filtered and concentrated. Flash chromatography $\left(500 \mathrm{~g} \mathrm{SiO}_{2}, \mathrm{CH}_{2} \mathrm{Cl}_{2}: \mathrm{MeOH}\right.$ : aqueous ammonia $=96: 3.5: 0.5)$ gave compound $7 \mathrm{a}$ as a colorless solid $(8.51 \mathrm{~g}, 61 \%) .{ }^{1} \mathrm{H}$ $\operatorname{NMR}\left(\mathrm{CDCl}_{3}\right) \delta 6.85(\mathrm{~s}, 1 \mathrm{H}), 4.56(\mathrm{~b}, 1 \mathrm{H}), 6.05-5.90(\mathrm{~m}, 2 \mathrm{H}), 4.48(\mathrm{~d}, 1 \mathrm{H}), 4.10(\mathrm{dd}, 1 \mathrm{H})$, $3.85(\mathrm{~d}, 1 \mathrm{H}), 3.80(\mathrm{~s}, 3 \mathrm{H}), 3.35-3.05(\mathrm{~m}, 2 \mathrm{H}), 2.62(\mathrm{~m}, 1 \mathrm{H}), 2.25(\mathrm{NH}, \mathrm{OH}), 1.98(\mathrm{~m}, 1 \mathrm{H})$, $1.85-1.65(\mathrm{~m}, 2 \mathrm{H}) ;{ }^{13} \mathrm{C} \mathrm{NMR}\left(\mathrm{CDCl}_{3}\right) \delta 145.8(\mathrm{~s}), 144.0(\mathrm{~s}), 134.1(\mathrm{~s}), 131.6(\mathrm{~s}), 127.9(\mathrm{~d})$, 126.8 (d), 115.5 (d), 113.0 (s), $88.4(\mathrm{~d}), 61.7$ (d), $56.0(\mathrm{q}), 52.7$ (t), 49.3 (s), 46.6 (t), 39.8 (t), 29.7 (t); TLC: $\mathrm{CH}_{2} \mathrm{Cl}_{2}: \mathrm{MeOH}=9: 1, \mathrm{R}_{\mathrm{f}}=0.65$; m. p.: $152-153{ }^{\circ} \mathrm{C}$.

(6R)-3-Methoxy-5,6,9,10,11,12-hexahydro-4aH-benzofuro[3a,3,2-ef][2]benzazepin-6-ol (7b). A suspension of zinc dust (19.0 g, $290 \mathrm{mmol})$ and copper(I)iodide (19.0 g, $100 \mathrm{mmol})$ in ethanol $(200 \mathrm{~mL}) /$ water $(200 \mathrm{~mL})$ was sonicated for $1 \mathrm{~h}$ under an argon atmosphere. Calcium chloride $(14.0 \mathrm{~g}, 126 \mathrm{mmol})$ and $7 \mathrm{a}(7.0 \mathrm{~g}, 19.9 \mathrm{mmol})$ were added, and the mixture was refluxed for $3 \mathrm{~h}$, filtered and concentrated. The residue was dissolved in $\mathrm{CHCl}_{3}(100 \mathrm{~mL})$ and washed with water $(1 \times 100 \mathrm{~mL})$. The aqueous layer was extracted with $\mathrm{CHCl}_{3}(2 \times 100 \mathrm{~mL})$, the combined organic layers were washed water $(3 \times 100 \mathrm{~mL})$, brine $(2 \times 100 \mathrm{~mL})$, dried $\left(\mathrm{Na}_{2} \mathrm{SO}_{4}\right)$, filtered and concentrated. The cristalline residue was washed with diisopropyl ether $(50 \mathrm{~mL})$ to give the debrominated compound $7 \mathbf{b}(4.45 \mathrm{~g}, 82 \%) .{ }^{1} \mathrm{H} \mathrm{NMR}\left(\mathrm{CDCl}_{3}\right): \delta 6.65-6.52(\mathrm{~m}, 2 \mathrm{H}), 6.06-5.92$ (m, 2 H), 4.57 (b, $1 \mathrm{H}), 4.15-4.08(\mathrm{~m}, 1 \mathrm{H}), 3.95$ (d, J = 5.7 Hz, $2 \mathrm{H}), 3.79$ (s, $3 \mathrm{H}), 3.34$ (ddd, $J$ $=14.6,3.5,3.5 \mathrm{~Hz}, 1 \mathrm{H}), 3.18(\mathrm{ddd}, J=13.2,11.4,2.6 \mathrm{~Hz}, 1 \mathrm{H}), 2.66(\mathrm{ddd}, J=15.7,1.63,1.63$ $\mathrm{Hz}, 1 \mathrm{H}), 1.98(\mathrm{ddd}, J=15.7,5.0,2.4 \mathrm{~Hz}, 1 \mathrm{H}), 1.88-1.61(\mathrm{~m}, 2 \mathrm{H}) ;{ }^{13} \mathrm{C} \mathrm{NMR}\left(\mathrm{CDCl}_{3}\right): \delta 146.2$ (s), 143.9 (s), 133.1 (s), 133.0 (s), 127.6 (d), 127.0 (d), 120.5 (d), 111.0 (d), 88.5 (d), 61.9 (d), $55.8(\mathrm{q}), 53.8(\mathrm{t}), 48.7(\mathrm{~s}), 47.0(\mathrm{t}), 40.3(\mathrm{t}), 29.9$ (t); TLC: $\mathrm{CHCl}_{3}: \mathrm{MeOH}=9: 1 ; \mathrm{R}_{\mathrm{f}}=0.35 ; \mathrm{m}$. p.: $233-235^{\circ} \mathrm{C}$. 
(6R)-3-Methoxy-5,6,9,10,11,12-hexahydro-6-hydroxy-4aH-benzofuro[3a,3,2ef][2]benzazepin-11-carboxylic acid ethyl ester (7c) Method A. A solution of norgalanthamine $7 \mathbf{b}(300 \mathrm{mg}, 1.10 \mathrm{mmol})$ and $\mathrm{Et}_{3} \mathrm{~N}(121 \mathrm{mg}, 1.20 \mathrm{mmol})$ in dry $\mathrm{CH}_{2} \mathrm{Cl}_{2}(5 \mathrm{~mL})$ was treated with ethyl chloroformate $(121 \mathrm{mg}, 1.12 \mathrm{mmol})$ in $\mathrm{CH}_{2} \mathrm{Cl}_{2}(1 \mathrm{~mL})$ at $0{ }^{\circ} \mathrm{C}$. The reaction mixture was stirred at this temperature for $1 \mathrm{~h}$, then $2 \mathrm{~N} \mathrm{HCl}(10 \mathrm{~mL})$ was added. The aqueous layer was extracted with $\mathrm{CH}_{2} \mathrm{Cl}_{2}(3 \times 3 \mathrm{~mL})$, the combined organic layers were washed with $2 \mathrm{~N} \mathrm{HCl}(2 \times 5 \mathrm{~mL})$, water $(1 \times 10 \mathrm{~mL})$, a saturated aqueous $\mathrm{NaHCO}_{3}$ solution $(2 \times 10 \mathrm{~mL})$, water $(1 \times 100 \mathrm{~mL})$, brine $(1 \times 10 \mathrm{~mL})$, dried $\left(\mathrm{Na}_{2} \mathrm{SO}_{4}\right)$, filtered and concentrated to give carbamate $7 \mathrm{c}$ as a colorless foam (349 $\mathrm{mg}, 92 \%)$.

Method B. To a suspension of carbamate $5 \mathbf{b}(100 \mathrm{mg}, 0.29 \mathrm{mmol})$ in dry THF $(4 \mathrm{~mL}) \mathrm{L}-$ Selectride ${ }^{\circledR}(1.46 \mathrm{~mL}, 1 \mathrm{~N}$ in THF, $1.46 \mathrm{mmol})$ was added at $-20^{\circ} \mathrm{C}$. The mixture was stirred for $2 \mathrm{~h}$ at this temperature, then water $(2 \mathrm{~mL})$ was added dropwise. After stirring for $10 \mathrm{~min}$. the mixture was concentrated, and the residue was dissolved in EtOAc $(10 \mathrm{~mL})$. The organic layer was washed with a saturated aqueous $\mathrm{NaHCO}_{3}$ solution $(1 \times 10 \mathrm{~mL})$, the aqueous layer was extracted with EtOAc $(2 \times 5 \mathrm{~mL})$. The combined organic layers were washed with a saturated aqueous $\mathrm{NaHCO}_{3}$ solution $(2 \times 10 \mathrm{~mL})$, water $(1 \times 10 \mathrm{~mL})$, brine $(1 \times 10 \mathrm{~mL})$, dried $\left(\mathrm{Na}_{2} \mathrm{SO}_{4}\right)$, filtered and concentrated. Flash chromatography $\left(10 \mathrm{~g} \mathrm{SiO}_{2}, \mathrm{CH}_{2} \mathrm{Cl}_{2}: \mathrm{MeOH}:\right.$ aqueous ammonia $=96: 3.5: 0.5)$ gave compound $7 \mathrm{c}$ as a colorless solid $(76.5 \mathrm{mg}, 76 \%) .{ }^{1} \mathrm{H}$ NMR $\left(\mathrm{CDCl}_{3}\right) \delta 6.82-6.56(\mathrm{~m}, 2 \mathrm{H}), 6.07-5.88(\mathrm{~m}, 2 \mathrm{H}), 4.99-4.60(\mathrm{~m}, 1 \mathrm{H}), 4.56(\mathrm{~s}, 1 \mathrm{H}), 4.38-$ 3.89 (m, 6 H), 3.78 (s, 3 H), $3.50-3.18(\mathrm{~m}, 1 \mathrm{H}), 2.62$ (dd, $1 \mathrm{H}), 2.40$ (s, $1 \mathrm{H}), 2.09-1.60$ (m, 3 $\mathrm{H}), 1.31-1.09(\mathrm{~m}, 3 \mathrm{H}) ;{ }^{13} \mathrm{C}$ NMR $\left(\mathrm{CDCl}_{3}\right) \delta 155.4$ and $155.3(\mathrm{~s}), 146.3(\mathrm{~s}), 144.1(\mathrm{~s}), 132.2$ and 131.9 (s), 129.2 (s), 127.9 (d), 126.2 (d), 121.3 and 120.7 (d), 111.1 and 110.8 (d), 88.1 and 88.0 (d), $61.6(\mathrm{~d}), 61.2(\mathrm{t}), 55.7(\mathrm{q}), 51.6$ and $51.2(\mathrm{t}), 48.2(\mathrm{~s}), 45.5$ and $45.2(\mathrm{t}), 37.1$ and $36.2(\mathrm{t})$, 29.7 (t), 14.4 and 14.3 (q); TLC: $\mathrm{CHCl}_{3}: \mathrm{MeOH}=9: 1, \mathrm{Rf}=0.75$; EtOAc, $\mathrm{R}_{\mathrm{f}}=0.65$.

\section{4a,5,9,10,11,12-hexahydro-3-methoxy-4'-methyl-spiro[6H-benzofuro[3a,3,2-}

ef][2]benzazepine-6,2'-[1,3]dioxolane]-11-carboxylic acid, ethyl ester (8). Keton $5 \mathrm{~b}$ (10.0 g, $29.1 \mathrm{mmol})$, p-toluene sulfonic acid $(150 \mathrm{mg})$ and 1,2-propanediol $(10 \mathrm{~mL})$ mixture were refluxed with stirring in toluene $(70 \mathrm{~mL})$ for 10 hours using a Dean-Stark type apparatus. A dropping funnel was charged with a solution of p-toluene sulfonic acid $(350 \mathrm{mg})$ in 1,2propanediol $(5 \mathrm{~mL})$. After the first hour of refluxing and then every $15 \mathrm{~min}$. for the remaining $5 \mathrm{~h}, 250 \mu \mathrm{L}$ of this solution were added to the refluxing mixture. The reaction mixture was cooled to $20^{\circ} \mathrm{C}$, and the lower phase was separated and extracted with toluene $(4 \times 10 \mathrm{~mL})$. The combined toluene phases were concentrated to a volume of $70 \mathrm{~mL}$. This solution then was cooled to $20^{\circ} \mathrm{C}$ and washed successively with $10 \%$ acetic acid $(20 \mathrm{~mL})$, saturated sodium hydrogen carbonate solution $(2 \times 20 \mathrm{~mL})$, water $(2 \times 20 \mathrm{~mL})$, brine $(20 \mathrm{~mL})$, dried $\left(\mathrm{Na}_{2} \mathrm{SO}\right)$, filtered and evaporated to dryness to yield the product $8(9.56 \mathrm{~g}, 82 \%)$ as a dry foam, which was directly used in the next step.

3-Methoxy-11-methyl-5,6,9,10,11,12-hexahydro-4aH-benzofuro[3a,3,2-ef][2]benzazepin-6one, ( \pm -narwedine $(( \pm)-9)$. To ketal $8(14.4 \mathrm{~g}, 35.9 \mathrm{~mol})$ in anhydrous THF $(30 \mathrm{~mL})$ a $10 \%$ solution of $\mathrm{LiAlH}_{4}$ in THF $(24 \mathrm{~mL}, 63.0 \mathrm{mmol})$ is added slowly with stirring to the suspension. 
Synthetic air ( $80 \%$ nitrogen, $20 \%$ oxygen) is introduced into the solution with stirring at 60 $65^{\circ} \mathrm{C}$ without external heating. The reaction is monitored by TLC and is allowed to continue until the starting material has been consumed completely (approximately $3 \mathrm{~h}$ ). Toluene $(20 \mathrm{~mL})$ is added, followed by dropwise addition of water $(2.5 \mathrm{~mL})$. During this decomposition a gel is formed intermittently, which is difficult to stir. Continued addition of water returns the mixture to a stirrable suspension. At this stage $15 \%$ sodium hydroxide $(2.5 \mathrm{~mL})$ is added rapidly and stirred for $15 \mathrm{~min}$. followed by the addition of diatomaceous earth $(2.5 \mathrm{~g})$ as a filtration aid. The solution is stirred for $30 \mathrm{~min}$ at reflux and filtered while hot. The precipitate is hot-extracted with toluene $:$ THF $=1: 1(3 \times 20 \mathrm{~mL})$ and the combined filtrates are evaporated to dryness. The crude product is deprotected by adding $4 \mathrm{~N} \mathrm{HCl}(25 \mathrm{~mL})$ and stirring for $20 \mathrm{~min}$ at $60^{\circ} \mathrm{C}$. After adjusting the $\mathrm{pH}$ of the solution to $<1$ by addition of $4 \mathrm{~N} \mathrm{HCl}$ the solution is cooled and washed with EtOAc $(2 \times 15 \mathrm{~mL})$. The acidic, aqueous phase is concentrated by vacuum distillation $\left(40^{\circ} \mathrm{C}, 20-30 \mathrm{mbar}\right)$ to a volume of $15 \mathrm{~mL}$. To the residue $25 \%$ ammonium hydroxide $(20 \mathrm{~mL})$ and ice $(10 \mathrm{~g})$ are added, the suspension of crystalline material is stirred for $30 \mathrm{~min}$, filtered, washed with water $(3 \times 5 \mathrm{~mL})$ and dried at $70^{\circ} \mathrm{C}$ and 40 mbar to give the product $( \pm)-9(8.09 \mathrm{~g}$, $79 \%$ ) as white to pale yellow powder. IR (KBr) 3014, 2919, 2844, 1681, 1618, 1587, 1540, $1505,1440,1285,1265,1212,1167,1146,1133,1102,1050,1029,1000 \mathrm{~cm}^{-1} ;{ }^{1} \mathrm{H}$ NMR $\left(\mathrm{CDCl}_{3}\right) \delta 6.93(\mathrm{~d}, 1 \mathrm{H}, J=12.8 \mathrm{~Hz}), 6.64(\mathrm{AB}, 2 \mathrm{H}, J=8.5 \mathrm{~Hz}), 6.00(\mathrm{~d}, 1 \mathrm{H}, J=12.8 \mathrm{~Hz}), 4.68$ $(\mathrm{m}, 1 \mathrm{H}), 4.06(\mathrm{~d}, 1 \mathrm{H}, J=16.0 \mathrm{~Hz}), 3.80(\mathrm{~s}, 3 \mathrm{H}), 3.70(\mathrm{~d}, 1 \mathrm{H}, J=16.0 \mathrm{~Hz}), 3.02-3.28(\mathrm{~m}, 3$ H), $2.71(\mathrm{dd}, 1 \mathrm{H}, J=19.2 \mathrm{~Hz}, J=3.2 \mathrm{~Hz}), 2.41(\mathrm{~s}, 3 \mathrm{H}), 2.15-2.30(\mathrm{~m}, 1 \mathrm{H}), 1.75-1.90(\mathrm{~m}, 1$ $\mathrm{H}) ;{ }^{13} \mathrm{C} \mathrm{NMR}\left(\mathrm{CDCl}_{3}\right) \delta 194.4,147.0,144.4,144.0,130.6,129.4,127.1,122.0,111.9,88.0,60.7$, 56.0, 54.1, 49.0, 42.5, 37.3, 33.3; TLC: $\mathrm{CHCl}_{3}: \mathrm{MeOH}=95: 5, \mathrm{R}_{\mathrm{f}}=0.45$; m. p.: $191-193^{\circ} \mathrm{C}$.

(-)-Narwedine ((-)-9). $( \pm)-9(7.0 \mathrm{~g})$ in ethanol : triethylamine $=9: 1(75 \mathrm{~mL})$ is heated to reflux and ethanol : triethylamine $=9: 1(10 \mathrm{~mL})$ is added to give a homogeneous solution. The solution is cooled to $65-68^{\circ} \mathrm{C}$, seeded with (-)-9 $(70 \mathrm{mg})$ cooled to $40^{\circ} \mathrm{C}$ within $1 \mathrm{~h}$ and stirred for $3 \mathrm{~h}$. The suspension is concentrated at $40^{\circ} \mathrm{C}$ and $20-40$ mbar to a volume of $30 \mathrm{~mL}$, cooled to $5-10^{\circ} \mathrm{C}$ with stirring, filtered and washed with cold ethanol $(4 \mathrm{~mL})$. After drying at $60^{\circ} \mathrm{C}$ and 40 mbar, $5.61 \mathrm{~g}(80 \%)$ of $(-)-9$ is recovered. $\alpha=-400, \mathrm{c}=1.5 \%$ in $\mathrm{CHCl}^{20}{ }_{3}$, Lit. $^{7}: \alpha_{20}=-405 .{ }^{D}$

\section{5,6,9,10,11,12-Hexahydro-3-methoxy-6-oxo-4aH-benzofuro[3a,3,2-ef][2]benzazepin-11-}

carboxylic acid ethyl ester (5b). To a solution of oxalyl chloride $(110 \mathrm{mg}, 0.87 \mathrm{mmol})$ in dry $\mathrm{CH}_{2} \mathrm{Cl}_{2}(3 \mathrm{~mL})$, DMSO $(68 \mathrm{mg}, 0.87 \mathrm{mmol})$ in dry $\mathrm{CH}_{2} \mathrm{Cl}_{2}(1 \mathrm{~mL})$ was added at $-78{ }^{\circ} \mathrm{C}$ and stirred for $1 \mathrm{~h}$ at this temperature. Carbamate $7 \mathrm{c}(150 \mathrm{mg}, 0.34 \mathrm{mmol})$ in dry $\mathrm{CH}_{2} \mathrm{Cl}_{2}(2 \mathrm{~mL})$ was added and stirred at $-60{ }^{\circ} \mathrm{C}$ for $5 \mathrm{~h}$. After addition of triethylamine $(200 \mathrm{mg}, 1.98 \mathrm{mmol})$ the mixture was stirred for $1 \mathrm{~h}$ at $-60{ }^{\circ} \mathrm{C}$ and $1 \mathrm{~h}$ at room temperature. The reaction mixture was washed with $2 \mathrm{~N} \mathrm{HCl}$, the aqueous layer was extracted with $\mathrm{CH}_{2} \mathrm{Cl}_{2}(2 \times 4 \mathrm{~mL})$, the combined organic layers were washed with $2 \mathrm{~N} \mathrm{HCl}(2 \times 10 \mathrm{~mL})$, a saturated aqueous $\mathrm{NaHCO}_{3}$ solution (2 x $10 \mathrm{~mL})$, water $(1 \times 10 \mathrm{~mL})$, brine $(1 \times 10 \mathrm{~mL})$, dried $\left(\mathrm{Na}_{2} \mathrm{SO}_{4}\right)$, filtered and concentrated to give keton $5 \mathbf{b}$ as a colorless solid (143 $\mathrm{mg}, 96 \%)$.

$( \pm)$-Galanthamine $(( \pm)-\mathbf{1})$. To a suspension of $\mathrm{LiAlH}_{4}(55 \mathrm{mg}, 1.44 \mathrm{mmol})$ in dry THF $(1 \mathrm{~mL})$ carbamate 7c $(100 \mathrm{mg}, 0.29 \mathrm{mmol})$ in dry THF $(0.5 \mathrm{~mL})$ was added dropwise. After stirring at 
reflux temperature for $12 \mathrm{~h}$ a saturated aqueous $\mathrm{NaHCO}_{3}$ solution $(3 \mathrm{~mL})$ was added, and the mixture was concentrated. The residue was dissolved in $\mathrm{CHCl}_{3}(5 \mathrm{~mL})$ and washed with a saturated aqueous $\mathrm{NaHCO}_{3}$ solution $(3 \times 3 \mathrm{~mL})$. The aqueous layer was extracted with $\mathrm{CHCl}_{3}(4$ $x 3 \mathrm{~mL}$ ). The combined organic layers were washed with a saturated aqueous $\mathrm{NaHCO}_{3}$ solution $(2 \times 5 \mathrm{~mL})$, water $(1 \times 5 \mathrm{~mL})$, brine $(1 \times 10 \mathrm{~mL})$, dried $\left(\mathrm{Na}_{2} \mathrm{SO}_{4}\right)$, filtered and concentrated. Flash chromatography $\left(10 \mathrm{~g} \mathrm{SiO}_{2}, \mathrm{CHCl}_{3}: \mathrm{MeOH}:\right.$ aqueous ammonia $\left.=97: 2.5: 0.5\right)$ gave compound 1 as a colorless solid (75 mg, 90\%), which was identical to galanthamine (HPLC, NMR).

(-)-Galanthamine ((-)-1). To a suspension of (-)-Narwedine ((-)-9) (1.0g, $3.50 \mathrm{mmol})$ in dry THF $(10 \mathrm{~mL})$ L-Selectride ${ }^{\circledR}\left(5.3 \mathrm{~mL}, 1 \mathrm{~N}\right.$ in THF) was added at $-20{ }^{\circ} \mathrm{C}$. The mixture was stirred for $2 \mathrm{~h}$ at this temperature, then water $(100 \mathrm{~mL})$ was added dropwise. After stirring for $10 \mathrm{~min}$. the mixture was concentrated, and the residue was dissolved in EtOAc $(20 \mathrm{~mL})$. The organic layer was washed with a saturated aqueous $\mathrm{NaHCO}_{3}$ solution $(1 \times 30 \mathrm{~mL})$, the aqueous layer was extracted with EtOAc $(2 \times 10 \mathrm{~mL})$. The combined organic layers were washed with a saturated aqueous $\mathrm{NaHCO}_{3}$ solution $(2 \times 30 \mathrm{~mL})$, water $(1 \times 10 \mathrm{~mL})$, brine $(1 \times 30 \mathrm{~mL})$, dried $\left(\mathrm{Na}_{2} \mathrm{SO}_{4}\right)$, filtered and concentrated. Flash chromatography $\left(50 \mathrm{~g} \mathrm{SiO}, \mathrm{CH}_{2} \mathrm{Cl}_{2}: \mathrm{MeOH}\right.$ : aqueous ammonia $=96: 3.5: 0.5)$ gave compound (-)-1 as a colorless solid (0.91 g, 91\%), which was identical to Galanthamine (HPLC, NMR). $\alpha=-95, \mathrm{c}=1 \%$ in $\mathrm{CHCl}^{25}{ }_{3}$, Lit. ${ }^{5}: \alpha_{25}=-93.4 D^{1} \mathrm{H}$ $\operatorname{NMR}\left(\mathrm{CDCl}_{3}\right) \delta 6.68-6.56(\mathrm{~m}, 2 \mathrm{H}), 6.08(\mathrm{~d}, 1 \mathrm{H}), 5.98(\mathrm{dd}, 1 \mathrm{H}), 4.59(\mathrm{~b}, 1 \mathrm{H}), 4.12(\mathrm{~b}, 1 \mathrm{H})$, 4.07 (d, 1 H), 3.66 (d, 1 H), 3.82 (s, 3 H), 2.38 (s, 3 H), 3.26 (ddd, 1 H), 3.03 (ddd, 1 H), 2.68 (ddd, $1 \mathrm{H}), 2.17$ - $1.92(\mathrm{~m}, 2 \mathrm{H}), 1.55$ (ddd, $1 \mathrm{H}) ;{ }^{13} \mathrm{C} \mathrm{NMR}\left(\mathrm{CDCl}_{3}\right) \delta 145.6(\mathrm{~s}), 143.8(\mathrm{~s}), 132.8$ (s), 129.2 (s), 127.4 (d), 111.0 (d), 126.7 (d), 121.8 (d), 88.4 (d), 61.8 (d), 60.4 (t), 55.6 (q), 41.9 $(\mathrm{q}), 53.6(\mathrm{t}), 48.0(\mathrm{~s}), 33.6(\mathrm{t}), 29.8(\mathrm{t})$.

\section{Acknowledgements}

The Institute of Organic Chemistry acknowledges financial support from Sanochemia Pharmazeutika AG.

\section{References and Notes}

1. Kueenburg, B.; Czollner, L.; Froehlich, J.; Jordis, U. Org. Process Res. Dev. 1999, 3, 425.

2. Sanchez, I. H.; Soria, J. J.; Lopez, F. J.; Larraza, M. I.; Flores, H. J. J. Org. Chem. 1984, 49, 157.

3. Czollner, L.; Frantsits, W.; Kueenburg, B.; Hedenig, U.; Froehlich, J.; Jordis, U. Tetrahedron Lett. 1998, 39, 2087.

4. Barton, D. H. R.; Kirby, G. W. J. Chem. Soc. 1962, 806.

5. Shieh, W.-O.; Carlson, J. A. J. Org. Chem. 1994, 59, 5463.

6. Koizumi, J.; Kobayashi, S.; Uyeo, S. Chem. Pharm. Bull. 1964, 12, 696.

7. Rizzi, A.; Schuh, R.; Bruckner, A.; Cvitkovich, B.; Kremser, L.; Jordis, U.; Froehlich, J.; Kueenburg, B.; Czollner, L. J. Chromatogr., B: Biomed. Sci. Appl. 1999, 730, 167. 